\title{
Phase II trial of dacomitinib in patients with HER2-positive gastric cancer
}

\author{
Do-Youn $\mathrm{Oh}^{1,2} \cdot$ Kewn-Wook Lee ${ }^{3} \cdot$ Jae Yong $\mathrm{Cho}^{4} \cdot$ Won Ki Kang ${ }^{5}$. \\ Seock-Ah Im ${ }^{1,2} \cdot$ Jin Won Kim ${ }^{3}$ Yung-Jue Bang ${ }^{1,2}$
}

Received: 30 September 2015/Accepted: 31 October 2015/Published online: 18 November 2015

(c) The International Gastric Cancer Association and The Japanese Gastric Cancer Association 2015

\begin{abstract}
Background Dacomitinib, an irreversible panHER inhibitor, shows significant preclinical antitumor activity in human epidermal growth factor receptor 2 (HER2)-positive gastric cancer (GC). The aim of this study was to evaluate the clinical activity of dacomitinib and discover potential biomarkers in HER2-positive GC patients.

Methods We enrolled previously treated advanced HER2-positive GC [HER2 FISH (+) or HER2 IHC 3+] patients. The patients received dacomitinib $45 \mathrm{mg}$ once daily.

Results A total of 27 patients were enrolled. The number of prior chemotherapy regimens was 1 in 7 patients (26\%), 2 in 9 patients (33\%), and more than 2 in 11 patients $(41 \%)$. Seven patients had received prior anti-HER2 therapy. The 4-month progression-free survival (PFS) rate was $22.2 \%$ and median PFS was 2.1 months (95\% CI, 2.3-3.4) There were 2 partial response (PRs) and 9 stable disease (SDs), resulting in $7.4 \%(95 \% \mathrm{CI}$,
\end{abstract}

A portion of this study was presented at the 2012 Gastrointestinal Cancer Symposium in San Francisco, CA, USA.

Yung-Jue Bang

bangyj@snu.ac.kr

1 Department of Internal Medicine, Seoul National University Hospital, Seoul National University College of Medicine, 101 Daehak-ro, Jongno-gu, Seoul 110-744, South Korea

2 Cancer Research Institute, Seoul National University College of Medicine, Seoul, South Korea

3 Department of Internal Medicine, Seoul National University Bundang Hospital, Seoul National University College of Medicine, Seoul, South Korea

4 Gangnam Severance Hospital, Seoul, South Korea

5 Samsung Medical Center, Seoul, South Korea
0-17.5\%) of response rate (RR) and $40.7 \%(95 \% \mathrm{CI}$, $21.9-59.6 \%$ ) of disease control rate (DCR). Eleven patients $(41 \%)$ showed some degree of tumor shrinkage. Overall survival was 7.1 months (95 \% CI, 4.4-9.8). The most common toxicities were skin rash, diarrhea, and fatigue, most of which were grade 1 or 2 . The $\mathrm{C}_{\text {trough }}$ of dacomitinib was lower in gastrectomy patients than nongastrectomy patients. Higher serum levels of HER2 extracellular domain (ECD) and lower levels of soluble E-cadherin (sECAD) correlated with higher dacomitinib activity.

Conclusions Dacomitinib functions as a single agent in HER2-positive GC patients with a tolerable safety profile. HER2 ECD and sECAD have the potential to be biomarkers for patient selection in a panHER inhibition strategy for HER2-positive GC. (ClinicalTrials.gov: NCT01152853).

Keywords HER2 - Gastric cancer - Dacomitinib - HER2 ECD $\cdot$ Soluble E-cadherin

\section{Introduction}

Gastric cancer (GC) is the fifth most common cancer and the third most common cause of cancer death worldwide [1]. It has been shown that chemotherapy prolongs overall survival (OS) of patients with advanced GC compared to best supportive care [2]. Combinations of fluoropyrimidines and platinum agents are the most widely used cytotoxic chemotherapy regimens [3]; however, the median OS of advanced GC remains approximately 1 year.

Human epidermal growth factor receptor 2 (HER2) is a member of the epidermal growth factor receptor (EGFR) family, which is involved in cancer cell proliferation, migration, and differentiation [4]. HER2 is overexpressed in 
20-25\% of breast cancers and is related to aggressive tumor biology and poor patient outcomes. Targeting HER2 in breast cancer has been very successful, and this approach has become the standard of care for HER2-positive breast cancer [5].

In gastric cancer, HER2 is overexpressed or amplified in $6-35 \%$ of cases $[6,7]$. To date, it is unclear whether HER2 positivity is associated with a poor prognosis in GC [8]. In HER2-positive advanced GC, the combination of trastuzumab with cisplatin and a fluoropyrimidine chemotherapy significantly improves OS statistically and clinically as compared with chemotherapy alone as first-line therapy [9], and it has become the standard of care. Even though HER2 positivity identifies patients who might obtain benefits from trastuzumab, HER2 overexpression alone does not adequately predict response to trastuzumab. Some HER2-positive GC patients show primary or secondary resistance to trastuzumab [10]. Lapatinib is a dual EGFR/HER2 tyrosine kinase inhibitor, and a combination of lapatinib and capecitabine has been reported to be effective in trastuzumab-resistant HER2-positive breast cancer patients $[11,12]$. In spite of this activity in breast cancer, a combination of lapatinib and chemotherapy in HER2-positive GC failed to show clinical activity in a secondline or even a first-line setting [13, 14].

Dacomitinib (PF-00299804), a panHER inhibitor, irreversibly binds to the adenosine triphosphate domain of each of the three kinase-active members of the HER family: HER1, HER2, and HER4. Dacomitinib inhibits proliferation of HER2-positive breast cancer cells resistant to trastuzumab or lapatinib [15]. Dacomitinib also shows a very strong preclinical antitumor efficacy in HER2-positive GC cells [16]. Neratinib, another panHER inhibitor, shows promising clinical activity as a single agent in HER2positive breast cancer patients who are pretreated with trastuzumab [17]. To date, there has been no report of the clinical activity of panHER inhibitor in HER2-positive GC patients.

Based on our preclinical study of dacomitinib in GC and clinical observation with neratinib in HER2-positive breast cancer, we hypothesized that dacomitinib might show clinical activity in HER2-positive GC patients as a single agent. Therefore, this study was conducted to evaluate the efficacy and safety of dacomitinib monotherapy in HER2positive GC patients. We also aimed to assess the pharmacokinetic variation of dacomitinib according to gastrectomy states and to elucidate the potential biomarkers for a panHER inhibition strategy in GC.

\section{Patients and methods}

This was a multicenter, single-arm, open-label phase II study. It was approved by the institutional review boards of all participating centers and was registered with the USA
National Library of Medicine (ClinicalTrials.gov) as NCT01152853. All studies were conducted according to guidelines (Declaration of Helsinki) for biomedical research.

\section{Patient eligibility criteria}

The following inclusion criteria were used for patient selection: (1) histologically confirmed adenocarcinoma of the stomach or gastroesophageal junction; (2) inoperable locally advanced or recurrent and/or metastatic disease, not amenable to curative therapy; (3) HER2 positivity defined as (a) IHC (immunohistochemistry) 3+ and/or (b) HER2 FISH (fluorescence in situ hybridization) ( + , HER2/CEP17 $\geq 2.0)$; (4) failure of response to at least one chemotherapy regimen (trastuzumab or lapatinibpretreated patients were eligible); (5) measurable or evaluable disease based on the RECIST (Response Evaluation Criteria in Solid Tumors) criteria, version 1.1; (6) ECOG (Eastern Cooperative Oncology Group) performance status of $0-2$; and (7) adequate organ function including cardiac function [left ventricular ejection fraction (LVEF) $\geq 50 \%$ ]. Written informed consent was obtained from all participants. Important exclusion criteria were (1) known active brain metastases; (2) prior chemotherapy or surgery $<4$ weeks before study enrollment; (3) intestinal obstruction or impending obstruction; (4) cardiac arrhythmia (grade $\geq 2$ ), atrial fibrillation of any grade, or a QTc interval $>470 \mathrm{~ms}$.

\section{Treatment}

Dacomitinib was given at a dose of $45 \mathrm{mg}$ daily without interruption. The cycles were repeated every 28 days. The next cycle was delayed until the following criteria were satisfied: $\mathrm{ANC}>1,000$ cells $/ \mu \mathrm{l}$, platelet count $>75,000$ cells $/ \mu \mathrm{l}$, and nonhematological toxicity related to dacomitinib returning to baseline levels or grade 1 or below. Dacomitinib administration was interrupted for patients with grade 3 hematological or nonhematological toxicity and resumed at one dose level lower after toxicity was reduced to grade 1 . Dose modifications were performed in response to adverse events where lower dose levels included 30 and $15 \mathrm{mg}$ daily, subsequently. Dose reduction was required for patients with grade 2 pneumonitis. Dacomitinib was permanently discontinued if a patient was unable to tolerate the drug at a dose of $15 \mathrm{mg}$ daily or if drug-related toxicity required treatment interruption for $>4$ weeks. Treatment was discontinued for patients with progressive disease, individuals who experienced unacceptable adverse events, or patients who withdrew consent. 


\section{Assessment of therapeutic efficacy and toxicity}

Tumor responses were assessed every 8 weeks or earlier in patients with suspected progression, based on the RECIST criteria (version 1.1). Computed tomography (CT) scans were conducted at least 4 weeks after the first scan to confirm complete or partial responses. The primary endpoint for measuring treatment efficacy was the 4-month PFS rate (4-month PFSR). PFS was defined as the time between the start of treatment and disease progression or death from any cause. Secondary endpoints for evaluating efficacy included response rates, disease control rates, duration of response, and OS. Overall survival was estimated from the date of the first treatment to patient death or the date of the last follow-up visit. Safety was assessed according to the NCI-CTCAE (Common Terminology Criteria for Adverse Events) (version 4.0).

\section{Statistical analysis}

The main purpose of this study was to assess the 4-month PFSR. The H0 (null hypothesis) expected a 4-month PFSR of $20 \%$ or less, and the H1 (alternative hypothesis) expected a 4-month PFSR of $35 \%$ or more. The rationale for this hypothesis was based on our previous study of advanced gastric cancer patients. The PFS of first-line chemotherapy, second-line chemotherapy, third-line chemotherapy, and fourth-line chemotherapy were 4.36 months $(95 \% \mathrm{CI}$, 4.03-4.71), 2.67 months (95\% CI, 2.32-3.01), 2.57 months (95\% CI, 2.25-2.88), and 2.17 months (95\% CI, 1.77-2.56) respectively. Because most of the patients eligible for this current study had a high probability of pretreatment with one or two kinds of chemotherapies, we set the $\mathrm{H} 0$ as $20 \%$ 4-month PFSR. As we expected that dacomitinib could improve the 4-month PFSR by $75 \%$, we set the H1 as 4-month PFSR of $35 \%$. To test these hypotheses, 25 evaluable patients were required to provide $80 \%$ power with a onesided significance level of 0.2 , and assuming a $15 \%$ dropout rate, an enrollment of 28 patients was required. The intentionto-treat population was defined as the population that included patients who were dosed at least once. Per protocol population was defined as the population who received the dacomitinib for at least 8 weeks. Survival and all safety analyses were carried out on the intention-to-treat population. The objective response was assessed on a per protocol population. OS and PFS were analyzed using the Kaplan-Meier method. The survival results were expressed as the median value with a $95 \%$ confidence interval (CI).

\section{Pharmacokinetics assessment}

We measured the plasma concentration of dacomitinib at baseline (within 7 days before treatment initiation), C1D14 (cycle 1, day 14), and at C2D1 and C2D14, before the intake of dacomitinib. Plasma dacomitinib concentrations were determined using a validated, sensitive high-performance liquid chromatography-atomic pressure ionization tandem mass spectrometric method in compliance with Pfizer Standard Operating Procedures.

\section{Biomarker assessment}

We collected blood samples from all patients for biomarker analysis at baseline, C2D1, and end of treatment (EOT). Quantitation of HER2 extracellular domain (HER2 ECD) and soluble E-cadherin (sECAD) in serum was conducted by sandwich enzyme-linked immunosorbent assay (ELISA)

Table 1 Patient characteristics $(n=27)$

\begin{tabular}{|c|c|c|}
\hline Characteristic & Number & Percent \\
\hline \multicolumn{3}{|l|}{ Age (years) } \\
\hline Median & 61 & $(43-80)$ \\
\hline \multicolumn{3}{|l|}{ Gender } \\
\hline M & 22 & $81.5 \%$ \\
\hline $\mathrm{F}$ & 5 & $18.5 \%$ \\
\hline \multicolumn{3}{|l|}{ Differentiation } \\
\hline Well-differentiated & 1 & $3.7 \%$ \\
\hline Moderately differentiated & 11 & $40.7 \%$ \\
\hline Poorly differentiated & 6 & $22.2 \%$ \\
\hline Unknown & 9 & $33.3 \%$ \\
\hline \multicolumn{3}{|l|}{ Performance status } \\
\hline ECOG 0 & 9 & $33.3 \%$ \\
\hline ECOG 1 & 16 & $59.3 \%$ \\
\hline ECOG 2 & 2 & $7.4 \%$ \\
\hline \multicolumn{3}{|l|}{ Primary tumor site } \\
\hline Gastric & 26 & $96.3 \%$ \\
\hline Gastroesophageal junction & 1 & $3.7 \%$ \\
\hline \multicolumn{3}{|l|}{ HER2 status } \\
\hline IHC3+ & 17 & $63.0 \%$ \\
\hline IHC2+/FISH+ & 8 & $29.6 \%$ \\
\hline IHC1+/FISH+ & 2 & $7.4 \%$ \\
\hline \multicolumn{3}{|c|}{ Previous chemotherapy sessions } \\
\hline 1 & 5 & $18.5 \%$ \\
\hline 2 & 10 & $37.0 \%$ \\
\hline 3 & 7 & $25.9 \%$ \\
\hline More than 3 & 5 & $18.5 \%$ \\
\hline \multicolumn{3}{|c|}{ Previous exposure to HER2 targeting agent } \\
\hline Trastuzumab & 2 & $7.4 \%$ \\
\hline Lapatinib & 5 & $18.5 \%$ \\
\hline None & 20 & $74.1 \%$ \\
\hline \multicolumn{3}{|l|}{ Previous gastrectomy } \\
\hline Total gastrectomy & 4 & $14.8 \%$ \\
\hline Subtotal gastrectomy & 9 & $33.3 \%$ \\
\hline None & 14 & $51.9 \%$ \\
\hline
\end{tabular}


Fig. 1 Waterfall plot showing the maximum reduction of target lesion. $P D$ progressive disease

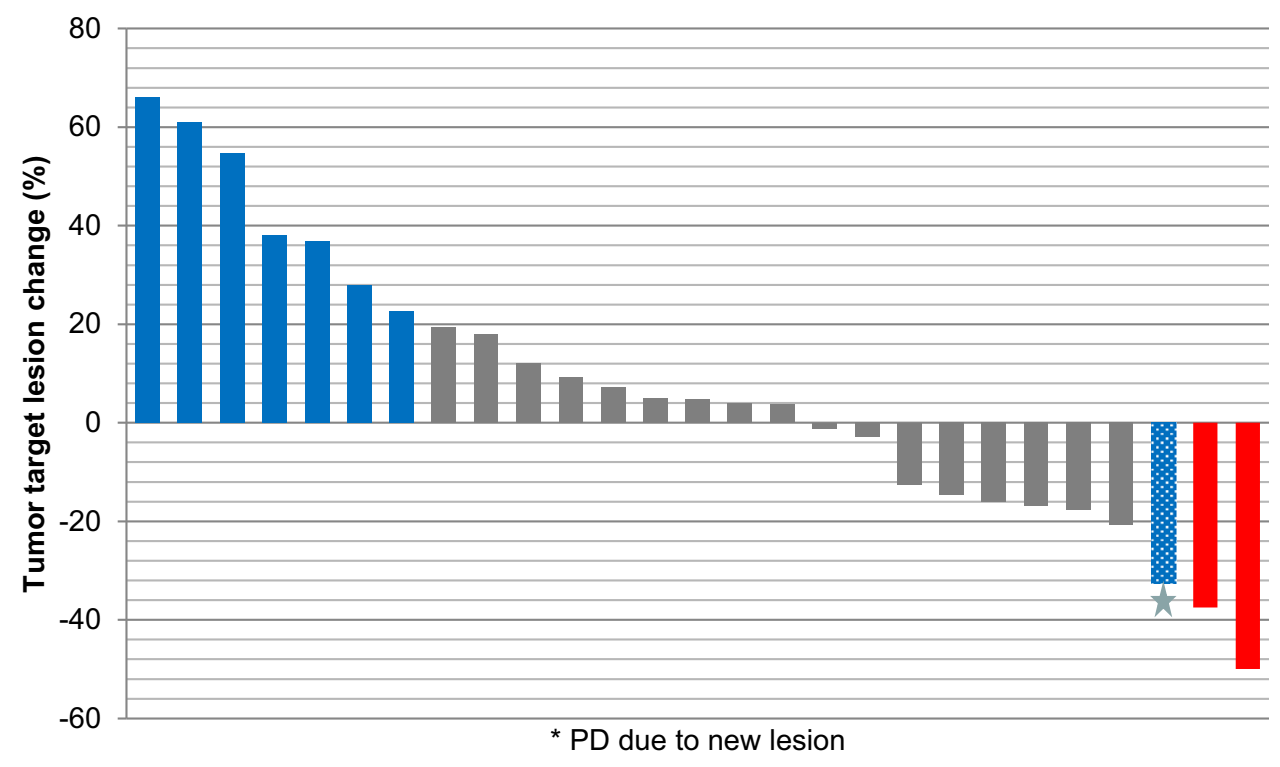

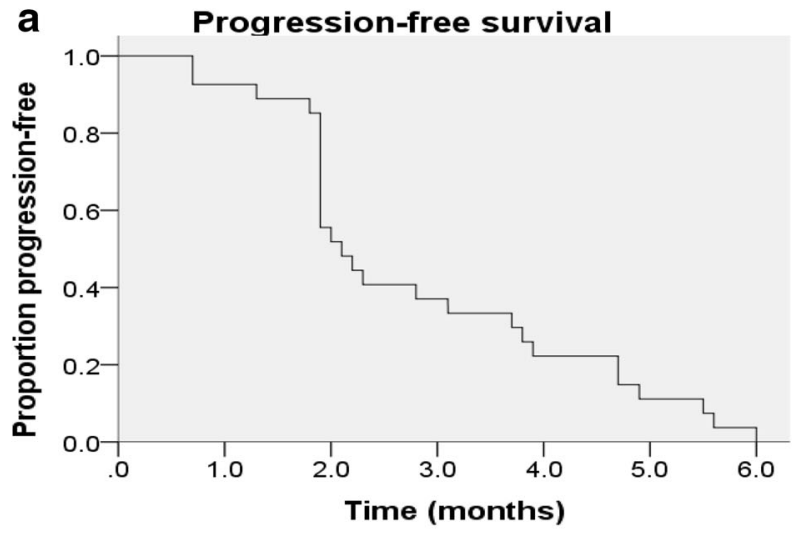

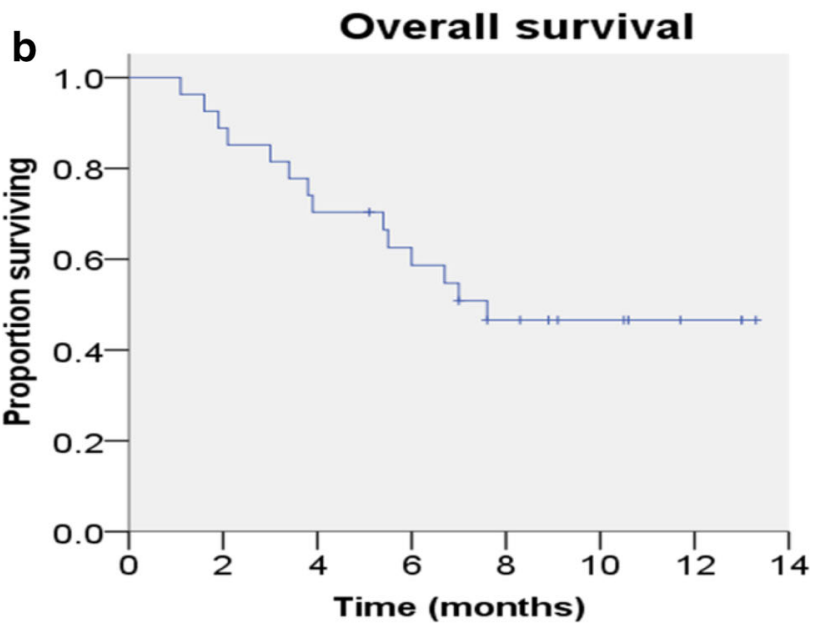

Fig. 2 Progression-free survival (a) and overall survival (b)

using a HER-2/neu ELISA Kit (Wilex, \#64898760) and a Human sE-Cadherin ELISA Kit ( $R$ \& D Systems, \#DCADE0), respectively.

\section{Results}

\section{Patient characteristics}

From November 2010 to April 2011, 28 patients were enrolled in the study. One patient withdrew his informed consent before treatment and 27 patients underwent dacomitinib treatment. The median age was 61 years (range, 43-80 years); 9 patients had ECOG performance status 0 and 16 patients had ECOG 1 . The number of previous chemotherapy exposures was 1 in 5 patients (19\%), 2 in 10 patients $(37 \%), 3$ in 7 patients (26\%), and $>3$ in 5 patients $(19 \%)$ (Table 1$)$.

The HER2 status was IHC3+ in 17 patients $(63 \%)$, $\mathrm{IHC} 2+/ \mathrm{FISH}+$ in 8 patients $(30 \%)$ and $\mathrm{IHC} 1+/ \mathrm{FISH}+$ in 2 patients $(7 \%)$. Seven patients were exposed to prior antiHER2 treatment (2 trastuzumab, 5 lapatinib). Thirteen patients had undergone prior gastrectomy (4 total gastrectomies and 9 subtotal gastrectomies). Safety and tumor response were evaluated in all 27 patients.

The median follow-up time period was 7.1 months (95\% CI, 5.6-9.6 months). All patients discontinued the treatment because of disease progression. A total of 84 cycles of treatment were delivered to the patients. The median number of cycles per patient was 2 (range, 1-6 cycles).

\section{Efficacy}

Partial response was observed in 2 patients, and 9 patients showed stable disease. The overall response rate was $7 \%$ (95\% CI, 0-17.5\%), and the disease control rate was $40.7 \%$ (95 \% CI, 21.9-59.6 \%) (Supplementary Table 1). Eleven patients $(41 \%)$ showed some degree of tumor 
Table 2 Adverse events $(n=27)$

\begin{tabular}{lcl}
\hline & $\begin{array}{l}\text { All grades } \\
\text { Number of patients (\%) }\end{array}$ & $\begin{array}{l}\text { Grade 3-4 } \\
\text { Number of patients (\%) }\end{array}$ \\
\hline Rash & $20(74.1)$ & $2(7.4)$ \\
Diarrhea & $18(66.7)$ & $1(3.7)$ \\
Hypoglycemia & $1(3.7)$ & $1(3.7)$ \\
Hyponatremia & $1(3.7)$ & $1(3.7)$ \\
Ileus & $1(3.7)$ & $1(3.7)$ \\
Dysphagia & $1(3.7)$ & $1(3.7)$ \\
Fatigue & $10(37.0)$ & $0(0)$ \\
Fissure (hand, foot) & $8(29.6)$ & $0(0)$ \\
Paronychia & $4(14.8)$ & $0(0)$ \\
Nausea/vomiting & $7(25.9)$ & $0(0)$ \\
Stomatitis & $7(25.9)$ & $0(0)$ \\
Anorexia & $6(22.2)$ & $0(0)$ \\
Itching & $4(14.8)$ & $0(0)$ \\
Abdominal pain & $4(14.8)$ & $0(0)$ \\
\hline
\end{tabular}

shrinkage (Fig. 1). Among these 11, 1 patient showed disease progression because of a new lesion despite the shrinkage of target lesions. The median PFS was 2.1 months (95\% CI, 2.3-3.4 months), and the median OS was 7.1 months (95\% CI, 4.4-9.8 months) (Fig. 2). The 4-month PFSR was $22.2 \%$. Clinical efficacy did not differ by age, performance status, number of previous chemotherapy treatments, HER2 status, or prior anti-HER2 treatment (Supplementary Table 2). The 4-month PFSR was $25 \%$ in patients with prior exposure to anti-HER2 treatment, and that of anti-HER2 treatment-naive patients was $21.1 \%$ (HR 1.7, $p=0.711)$.

\section{Safety}

The most common adverse events (AEs; all grades) were rash $(74 \%)$, diarrhea $(67 \%)$, fatigue $(37 \%)$, stomatitis (26\%), and nausea (26\%) (Table 2). The grade 3/4 AEs were rash $(7 \%)$, diarrhea (4\%), hypoglycemia $(4 \%)$, and hyponatremia $(4 \%)$. There were no treatment-related deaths.

\section{Pharmacokinetics}

Observed trough plasma concentration $\left(\mathrm{C}_{\text {trough }}\right)$ ranged from 20 to $96 \mathrm{ng} / \mathrm{ml}$ on C1D14, 10 to $105 \mathrm{ng} / \mathrm{ml}$ on C2D1, and 15 to $100 \mathrm{ng} / \mathrm{ml}$ on C2D14. The $\mathrm{C}_{\text {trough }}$ of patients with gastrectomy was lower than that of patients without gastrectomy (Fig. 3).

\section{Biomarker analysis}

The baseline serum level of HER2 ECD correlated well with the HER2 expression in tumor tissue (Fig. 4a). The baseline mean level of HER2 ECD was highest in patients with a PR response, which was followed by SD with tumor shrinkage, SD without tumor shrinkage, and PD (Fig. 4b). In PR patients, the HER2 ECD level decreased on C2D1 and then increased dramatically at EOT (Fig. 4c). In contrast to the HER2 ECD, the baseline level of sECAD (soluble E-cadherin) was highest in patients with a PD response, which was followed by $\mathrm{SD}$ without tumor shrinkage, SD with tumor shrinkage, and PR patients (Fig. 4b). When we consider the baseline levels of both HER2 ECD and sECAD, patients with low-HER2 ECD and high sECAD did not show any tumor shrinkage (Fig. 4d). In PR patients, the sECAD level decreased on C2D1 and then increased at EOT (Fig. 4e).

\section{Discussion}

We tested dacomitinib monotherapy in HER2-positive GC patients who failed to respond to prior chemotherapies. Most $(82 \%)$ of the study population received dacomitinib as a third-line or later treatment setting, with $44 \%$ of the study population treated with dacomitinib as a fourth line and beyond. Considering the population characteristics, the PFS of 2.1 months and OS of 7.1 months are acceptable. Interestingly, with dacomitinib monotherapy, two patients showed PR, $41 \%$ of the patients showed some degree of tumor shrinkage, and $41 \%$ showed a disease control rate $(\mathrm{PR}+\mathrm{SD})$. With lapatinib monotherapy, when administered to the previously treated HER2-positive GC patients, there were no cases of PR; the disease control rate was only $10 \%$, the median PFS was 1.3 months $(95 \% \mathrm{CI}$, 1.2-1.7 months), and the median OS was 4.7 months (95\% CI, 1.8 months-NA), which led to the conclusion 

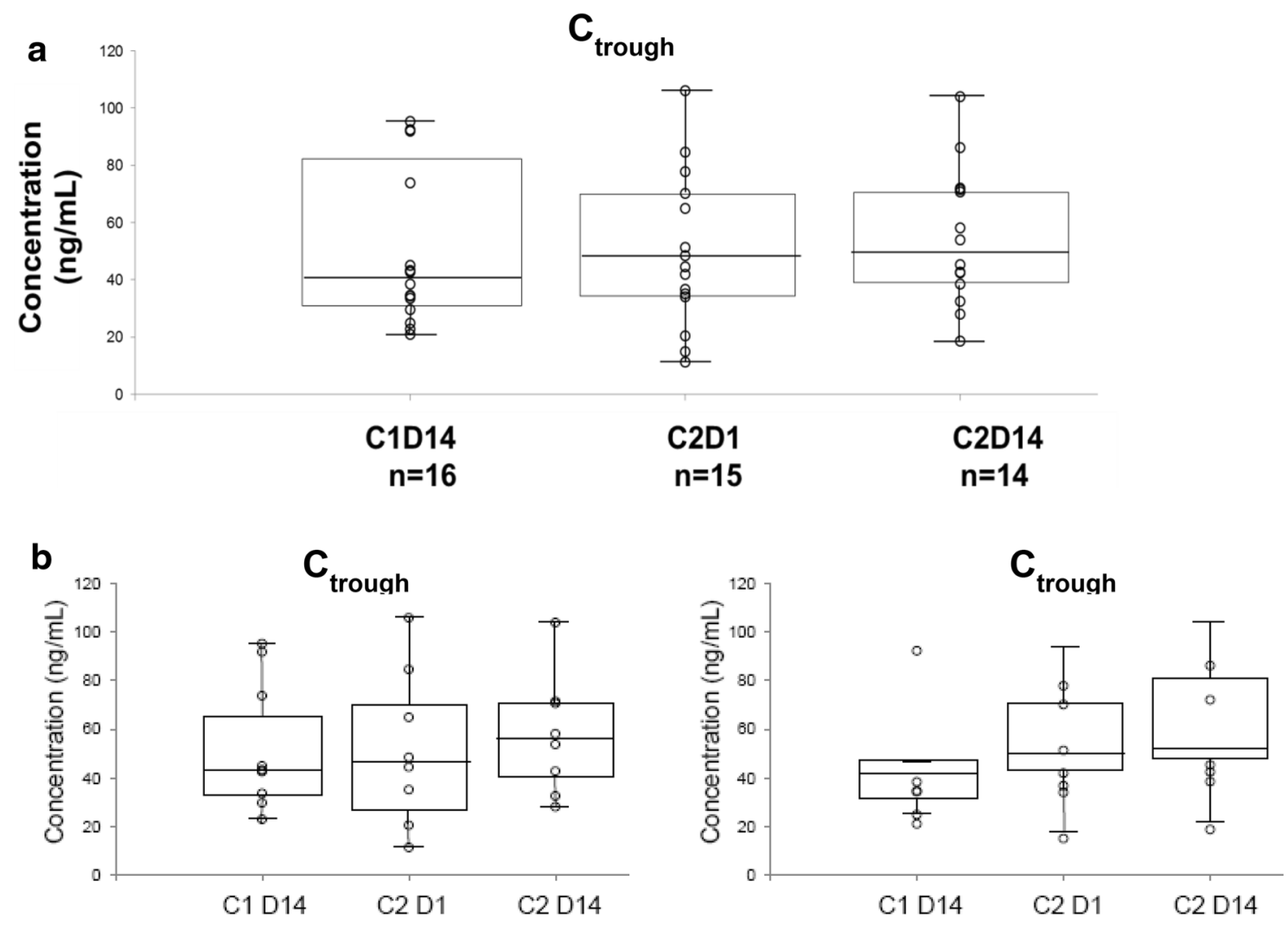

Without gastrectomy

With gastrectomy

\begin{tabular}{|l|c|c|c|c|c|c|}
\hline & \multicolumn{2}{|c|}{ Total } & \multicolumn{2}{c|}{ Without gastrectomy } & \multicolumn{2}{|c|}{ With gastrectomy } \\
\hline Time point & $\mathrm{N}$ & $\begin{array}{c}\text { Ctrough } \\
\text { Mean }(\% \mathrm{CV}) \\
{[\text { Median] }}\end{array}$ & $\mathrm{N}$ & $\begin{array}{c}\text { Ctrough } \\
\text { Mean }(\% \mathrm{CV}) \\
{[\text { Median] }}\end{array}$ & $\mathrm{N}$ & $\begin{array}{c}\text { Ctrough } \\
\text { Mean }(\% \mathrm{CV}) \\
{[\mathrm{Median}]}\end{array}$ \\
\hline Cycle 1, Day 14 & 16 & $47.9(53)[40.5]$ & 10 & $52.1(49)[43.2]$ & 6 & $40.9(64)[34.5]$ \\
\hline Cycle 2, Day 1 & 15 & $49.5(54)[44.5]$ & 8 & $52.0(62)[46.5]$ & 7 & $46.8(46)[42.0]$ \\
\hline Cycle 2 2 Day 14 & 14 & $54.7(44)[49.7]$ & 8 & $57.7(43)[56.0]$ & 6 & $50.6(48)[44.0]$ \\
\hline
\end{tabular}

$* \mathrm{CV}$ : Coefficient of variation

Fig. $3 \mathrm{C}_{\text {trough }}$ of dacomitinib (a) and $\mathrm{C}_{\text {trough }}$ according to gastrectomy status (b). a C $\mathrm{C}_{\text {trough }}$ of dacomitinib at C1D14 (cycle 1, day 14), C2D1, and C2D14. b C $\mathrm{C}_{\text {trough }}$ of dacomitinib at C1D14, C2D1, and C2D14

that lapatinib failed to show sufficient activity as a monotherapy in HER2-positive GC [18]. Taken together, the findings regarding the activity of dacomitinib monotherapy are encouraging in HER2-positive GC patients. This result of our hypothesis-generating small study provides evidence that the strategy of panHER inhibition might be feasible in HER2-positive GC patients, as is the case of neratinib in HER2-positive breast cancer cases [17].

The safety profile was consistent with a previous report using dacomitinib, and it was generally well tolerated [19]. according to gastrectomy status. The table summarizes the mean and median value of $\mathrm{C}_{\text {trough }}$ for all patients, patients with gastrectomy, and patients without gastrectomy

The pharmacokinetics (PKs) of dacomitinib have been evaluated in Western nation patients, Korean patients, and Japanese patients in previous phase I studies [20, 21]. The PK parameters of dacomitinib were similar across ethnicity). In our study, we conducted the PK evaluation because the literature contains no studies assessing whether a gastrectomy might affect the PKs of dacomitinib. In general, the $\mathrm{C}_{\text {trough }}$ of our study was slightly lower than that of Japanese patients, although the variation was similar [21]. Patients who had undergone a gastrectomy showed a lower level of $\mathrm{C}_{\text {trough }}$ than those who had not. These data are the 
Fig. 4 Analysis of biomarkers. a Correlation of human epidermal growth factor receptor 2 (HER2)

immunochemistry (IHC) and level of serum HER2

extracellular domain (ECD).

b Association of baseline levels of HER2 ECD and soluble E-cadherin (sECAD) with tumor response. $\mathrm{SD}+$ change indicates patients who show increase in tumor volume within the $\mathrm{SD}$ range; $\mathrm{SD}-$ change indicates patients who show decrease of tumor volume within the SD range. The baseline levels of HER2 ECD and SECAD were the mean value of each patient's levels in each group. $\mathbf{c}$ The change of HER2 ECD during treatment in partial response $(\mathrm{PR})$ patients. d Tumor response according to patient group relative to HER2 ECD and sECAD. Patients were divided into four groups according to HER2 ECD and sECAD: HER2 ECD high/ sECAD high, HER2 ECD high/ SECAD low, HER2 ECD low/ sECAD high, and HER2 ECD low/sECAD low. Tumor volume change of each patient in each group is shown. e Change of sECAD during treatment in PR patients a

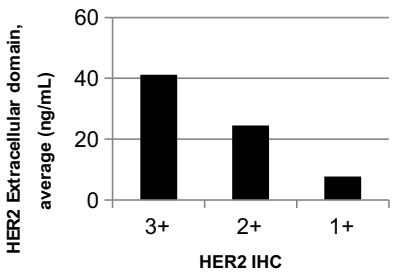

b
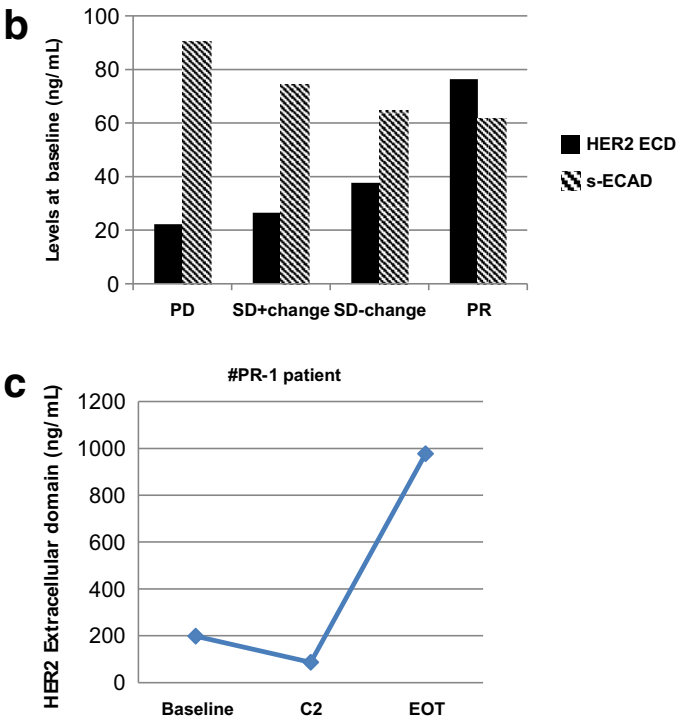

d
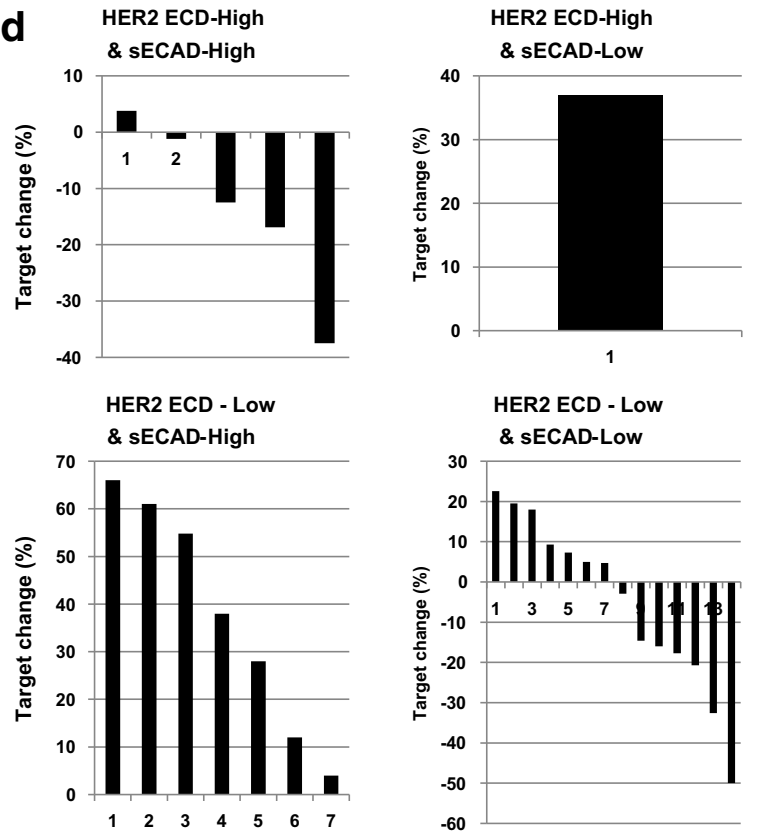

e
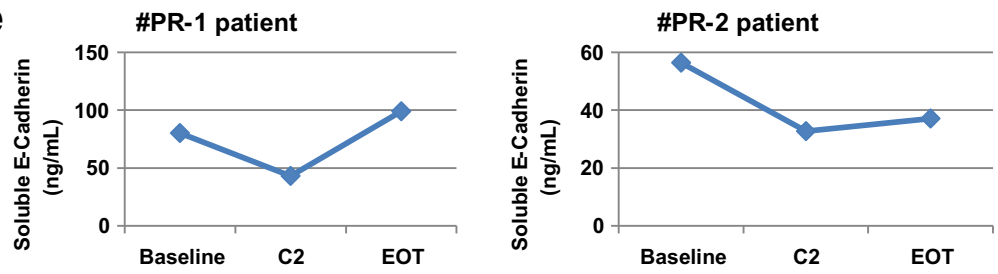
first PK data of dacomitinib focused on gastrectomy status. In previous PK studies, dacomitinib had an oral bioavailability of $80 \%$ following a $45-\mathrm{mg}$ dose and exhibited linear kinetics following single or multiple doses [20, 22]. Food has only a mild effect on absorption, but concomitant administration with antacids reduces absorption of dacomitinib [23]. This finding is consistent with our finding in gastrectomy patients because the gastric $\mathrm{pH}$ is increased by subtotal or total gastrectomy. Interindividual variability of dacomitinib is high (coefficient of variation up to $50 \%$ ), in part the result of the low solubility of dacomitinib (BCS class II compound with pH-dependent solubility) [23, 24].

To elucidate the candidate biomarkers for a panHER inhibitor in GC, we measured the serial serum levels of HER2 ECD and sECAD. Soluble truncated HER2 protein can be released through a proteolytic mechanism termed ECD shedding and also by alternative splicing of the HER2 transcript. Shedding of HER2 ECD significantly increased tyrosine kinase activity and considerably promoted the activity of $\mathrm{N}$-terminally truncated HER2 (p95 $\left.{ }^{\mathrm{HER} 2}\right)$, which is 10- to 100-fold more oncogenic than full-length HER2. Only a few studies have investigated the concordance between serum HER2 ECD level and tissue HER2 expression. In our study, the serum level of HER2 ECD correlated well with the level of HER2 protein expression in GC tumor tissue (Fig. 4a). Interestingly, the higher the baseline level of HER2 ECD, the more tumor shrinkage was obtained by dacomitinib (Fig. 4b). The predictive role of HER2 ECD for trastuzumab treatment has been debated in breast cancer [25] and has not been widely studied to date in GC [26]. Our study is the first report on the predictive role of HER2 ECD for panHER inhibitor treatment in GC. Furthermore, in our patients who showed PR, the level of HER2 ECD showed an early dramatic reduction at C2D1 and then increased dramatically at the time of disease progression. A similar observation was reported in a case of lung cancer treated with dacomitinib [27]. This finding suggests that measurement of serum HER2 ECD might give us predictive information regarding a panHER inhibitor in HER2-positive GC; however, this should be validated by further large-scale studies.

E-cadherin is a hemophilic adhesion molecule that is expressed at the basolateral membrane of epithelial tissues. The ectodermal shedding of a stable $80-\mathrm{kDa}$ sECAD has been shown to increase in the serum of cancer patients [28]; sECAD is disruptive to cell contact by inducing scattering and eroding the adherens junction by antagonizing full-length E-cadherin. EGF promotes the shedding of SECAD in an ADAM (a disintegrin and metalloprotease) family member-dependent manner, and this SECAD may act in EGFR signaling independently of traditional EGFR ligands $[29,30]$. Furthermore, in growth factor deprivation states, ADAM15 cleaves E-cadherin and this SECAD is found in complex with the HER2 and HER3 receptors.
These interactions contributed to stabilize HER2 heterodimerization [31]. We previously reported that dacomitinib acts to block the EGFR/HER2, HER2/HER3, and HER3/HER4 heterodimer formation, as well as the association of HER3 with $\mathrm{p} 85 \mathrm{a}^{16}$. In accordance with our preclinical finding, in this clinical study, when the patient's baseline serum level of sECAD was higher, less tumor shrinkage was obtained by dacomitinib (Fig. 4b).

When we take into consideration both HER2 ECD and sECAD, patients with HER2 ECD-low and sECAD-high did not show any tumor shrinkage (Fig. 4d). We suggest that these HER2 ECDs and SECADs might be candidates as patient selection biomarkers for dacomitinib treatment in HER2-positive GC. With a small sample size, it is difficult to make conclusive statements on the meaning of HER2 ECD and sECAD in HER2-positive GC. However, the findings observed in this study could provide information that is helpful for hypothesis generation for a future biomarker study in HER2-positive GC.

In conclusion, dacomitinib, an irreversible panHER inhibitor, functions as a single agent in HER2-positive GC patients and has an acceptable safety profile. HER2 ECD and SECAD have the potential to be biomarkers for patient selection in a panHER inhibition strategy for HER2-positive GC.

Acknowledgments We thank the patients included in the current study. We also thank Pfizer for the supply of dacomitinib.

Authors contribution Conception and design: D. Y. O., Y. J. B. Financial support and administrative support: D. Y. O., Y. J. B. Provision of study materials or patients: D. Y. O., K. W. L., J. Y. C., W. K. K., S. A. I., J. W. K., Y. J. B. Collection and assembly of data: D. Y. O., Y. J. B. Data analysis and interpretation: D. Y. O., Y. J. B. Drafting of the manuscript: D. Y. O., Y. J. B. Critical revision of the manuscript for technical content: D. Y. O., K. W. L., J. Y. C., W. K. K., S. A. I., J. W. K., Y. J. B. Final approval of manuscript: D. Y. O., K. W. L., J. Y. C., W. K. K., S. A. I., J. W. K., Y. J. B.

\section{Compliance with ethical standards}

Conflicts of interest Yung-Jue Bang: (1) consultant and advisory role: Pfizer; (2) honoraria: Pfizer; (3) research funding: Pfizer. None of the other investigators have any conflicts of interest to disclose.

Ethical standards All procedures followed were in accordance with the ethical standards of the responsible committee on human experimentation (institutional and national) and with the Helsinki Declaration of 1964 and later versions. Informed consent or substitute for it was obtained from all patients for being included in the study.

Funding sources This research was supported by Pfizer.

\section{References}

1. Ferlay J, Soerjomataram I, Dikshit R, et al. Cancer incidence and mortality worldwide: sources, methods and major patterns in GLOBOCAN 2012. Int J Cancer. 2015;136(5):E359-86. 
2. Wagner AD, Unverzagt S, Grothe W, et al. Chemotherapy for advanced gastric cancer. Cochrane Database Syst Rev. 2010;3:CD004064.

3. Garrido M, Fonseca PJ, Vieitez JM, et al. Challenges in first line chemotherapy and targeted therapy in advanced gastric cancer. Expert Rev Anticancer Ther. 2014;14(8):887-900.

4. Gravalos C, Jimeno A. HER2 in gastric cancer: a new prognostic factor and a novel therapeutic target. Ann Oncol. 2008;19:1523-9.

5. Arteaga CL, Sliwkowski MX, Osborne CK, et al. Treatment of HER2-positive breast cancer: current status and future perspectives. Nat Rev Clin Oncol. 2012;9:16-32.

6. Hofmann M, Stoss O, Shi D, et al. Assessment of a HER2 scoring system for gastric cancer: results from a validation study. Histopathology (Oxf). 2008;52:797-805.

7. Van Cutsem E, Bang YJ, Feng-Yi F, et al. HER2 screening data from ToGA: targeting HER2 in gastric and gastroesophageal junction cancer. Gastric Cancer. 2015;18(3):476-84.

8. Maresch J, Schoppmann SF, Thallinger CM, et al. Her-2/neu gene amplification and over-expression in stomach and esophageal adenocarcinoma: from pathology to treatment. Crit Rev Oncol Hematol. 2012;82:310-22.

9. Bang YJ, Van Cutsem E, Feyereislova A, et al. Trastuzumab in combination with chemotherapy versus chemotherapy alone for treatment of HER2-positive advanced gastric or gastro-oesophageal junction cancer (ToGA): a phase 3, open-label, randomised controlled trial. Lancet. 2010;376:687-97.

10. Ock CY, Lee KW, Kim JW, et al. Optimal patient selection for trastuzumab treatment in HER2-positive advanced gastric cancer. Clin Cancer Res. 2015;21(11):2520-9.

11. Geyer CE, Forster J, Lindquist D, et al. Lapatinib plus capecitabine for HER2-positive advanced breast cancer. N Engl J Med. 2006;355:2733-43.

12. Verma S, Miles D, Gianni L, et al. Trastuzumab emtansine for HER2-positive advanced breast cancer. $N$ Engl J Med. 2012;367(19):1783-91.

13. Satoh T, Xu RH, Chung HC, et al. Lapatinib plus paclitaxel versus paclitaxel alone in the second-line treatment of HER2amplified advanced gastric cancer in Asian populations: TyTANa randomized, phase III study. J Clin Oncol. 2014;32(19):2039-49.

14. Hecht J, Bang Y, Qin S, et al. Lapatinib in combination with capecitabine plus oxaliplatin (CapeOx) in HER2-positive advanced or metastatic gastric, esophageal, or gastroesophageal adenocarcinoma (AC): the TRIO-013/LOGiC trial ASCO Annual Meeting Abstracts Meeting Library. J Clin Oncol 2013;suppl:abstr LBA4001). http://meetinglibrary.asco.org/con tent/116873-132

15. Kalous O, Conklin D, Desai AJ, et al. Dacomitinib (PF00299804), an irreversible Pan-HER inhibitor, inhibits proliferation of HER2-amplified breast cancer cell lines resistant to trastuzumab and lapatinib. Mol Cancer Ther. 2012;11:1978-87.

16. Nam HJ, Ching KA, Kan J, et al. Evaluation of the antitumor effects and mechanisms of PF00299804, a pan-HER inhibitor, alone or in combination with chemotherapy or targeted agents in gastric cancer. Mol Cancer Ther. 2012;11:439-51.

17. Burstein HJ, Sun Y, Dirix LY, et al. Neratinib, an irreversible ErbB receptor tyrosine kinase inhibitor, in patients with advanced ErbB2-positive breast cancer. J Clin Oncol. 2010;28:1301-7.
18. Lorenzen S, Riera Knorrenschild J, Haag GM, et al. Lapatinib versus lapatinib plus capecitabine as second-line treatment in human epidermal growth factor receptor 2-amplified metastatic gastro-oesophageal cancer: a randomised phase II trial of the Arbeitsgemeinschaft Internistische Onkologie. Eur J Cancer. 2015;51(5):569-76.

19. Ramalingam SS, Blackhall F, Krzakowski M, et al. Randomized phase II study of dacomitinib (PF-00299804), an irreversible panhuman epidermal growth factor receptor inhibitor, versus erlotinib in patients with advanced non-small-cell lung cancer. J Clin Oncol. 2012;30:3337-44.

20. Janne PA, Boss DS, Camidge DR, et al. Phase I dose-escalation study of the pan-HER inhibitor, PF299804, in patients with advanced malignant solid tumors. Clin Cancer Res. 2011;17:1131-9.

21. Takahashi T, Boku N, Murakami H, et al. Phase I and pharmacokinetic study of dacomitinib (PF-00299804), an oral irreversible, small molecule inhibitor of human epidermal growth factor receptor-1, -2, and -4 tyrosine kinases, in Japanese patients with advanced solid tumors. Invest New Drugs. 2012;30(6):2352-63.

22. Giri N, LaBadie RR, Liang Y, et al. Absolute bioavailability of dacomitinib (PF-): Comparison of oral and intravenous administration in healthy volunteers. In: Proceedings of the 105th Annual Meeting of the American Association for Cancer Research; Apr 5-9, 2014, San Diego, CA. Abstract CT208.

23. Ruiz-Garcia A, Masters JC, LaBadie RR, et al. Effect of food and antacid treatment on bioavailability of $45 \mathrm{mg}$ tablet of dacomitinib relative to dacomitinib administration under fasted conditions (abstract PII-007). Clin Pharmacol Ther. 2014;95:S63.

24. Peters S, Zimmermann S, Adjei AA. Oral epidermal growth factor receptor tyrosine kinase inhibitors for the treatment of nonsmall cell lung cancer: comparative pharmacokinetics and drugdrug interactions. Cancer Treat Rev. 2014;40(8):917-26.

25. Lennon S, Barton C, Banken L, et al. Utility of serum HER2 extracellular domain assessment in clinical decision making: pooled analysis of four trials of trastuzumab in metastatic breast cancer. J Clin Oncol. 2009;27(10):1685-93.

26. Oyama K, Fushida S, Tsukada T, et al. Evaluation of serum HER2-ECD levels in patients with gastric cancer. J Gastroenterol. 2015;50(1):41-5.

27. Kelly RJ, Carter C, Giaccone G. Personalizing therapy in an epidermal growth factor receptor-tyrosine kinase inhibitor-resistant non-small-cell lung cancer using PF-00299804 and trastuzumab. J Clin Oncol. 2010;28(28):e507-10.

28. De Wever O, Derycke L, Hendrix A, et al. Soluble cadherins as cancer biomarkers. Clin Exp Metastasis. 2007;24:685-97.

29. Grabowska MM, Sandhu B, Day ML. EGF promotes the shedding of soluble E-cadherin in an ADAM10-dependent manner in prostate epithelial cells. Cell Signal. 2012;24:532-8.

30. Inge LJ, Barwe SP, D'Ambrosio J, et al. Soluble E-cadherin promotes cell survival by activating epidermal growth factor receptor. Exp Cell Res. 2011;317:838-48.

31. Najy AJ, Day KC, Day ML. The ectodomain shedding of E-cadherin by ADAM15 supports ErbB receptor activation. J Biol Chem. 2008;283:18393-401. 